\title{
Mechanism of Damped Oscillation in Microbubble Coalescence
}

\author{
Rou Chen ${ }^{\mathrm{a}}$, Jianhuan Zeng ${ }^{\mathrm{a}}$, Huidan(Whitney) $\mathrm{Yu}^{\mathrm{a}, *}$ \\ ${ }^{a}$ Department of Mechanical \& Energy Engineering, Indiana University-Purdue University Indianapolis, \\ Indianapolis (IUPUI), IN 46202, USA
}

\begin{abstract}
This work is part of our continuous research effort to reveal the underlying physics of bubble coalescence in microfluidics through the GPU-accelerated lattice Boltzmann method. We numerically explore the mechanism of damped oscillation in microbubble coalescence characterized by the Ohnesorge $(O h)$ number. The focus is to address when and how a damped oscillation occurs during a coalescence process. Sixteen cases with a range of $O h$ numbers from 0.039 to 1.543 , varying in liquid viscosity from 0.002 to $0.08 \mathrm{~kg} /(\mathrm{m} \cdot \mathrm{s})$ correspondingly, are systematically studied. First, a eriterion of with or without damped oscillation has been established. It is found that a larger $O h$ enables faster/slower bubble coalescence with/without damped oscillation when $(O h<0.477) /(O h>0.477)$ and the fastest coalescence falls at $O h \approx 0.477$. Second, the mechanism behind damped oscillation is explored in terms of the competition between driving and resisting forces. When $O h$ is small in the range of $O h<0.477$, the energy dissipation due to viscous effect is insignificant, sufficient surface energy initiates a strong inertia and overshoots the neck movement. It results in a successive energy transformation between surface energy and kinetic energy of the coalescing bubble. Through an analogy to the conventional damped harmonic oscillator, the saddle-point trajectory over the entire oscillation can be well predicted analytically.
\end{abstract}

Keywords:

microbubble coalescence, damped oscillation, damped harmonic oscillator, lattice

Boltzmann method, critical damping

\footnotetext{
*Corresponding author

Email address: whyu@iupui.edu (Huidan(Whitney) Yu)
} 


\section{Introduction}

Damped oscillation that switches the major axis between horizontal and vertical direction with reducing amplitude of the major/minor axis ratio is a unique behavior in microbubble coalescence. While forced oscillation is specifically induced by various external sources such as electrostatic force[1], acoustic trap[2], and laser light[3] for the purpose of controlling bubble size and collapse in different engineering and biomedical systems $[4,5,6]$, self-oscillation is inherently driven by imbalance between the driven and resistant forces. While the driving is from the surface tension at the gas-liquid interface, the resistance is co-contributed by inertial and viscous effects in the liquid side. Understanding the mechanism of self-oscillation is critically important to better utilize the forced oscillation in real-world applications. Rayleigh[7] was the pioneer who studied small-amplitude self-oscillation of an inviscid droplet from a pure mathematical point of view in 1879. Since then, Rayleigh's work has been extended toward physical environments by exploring the density effects of the surrounding fluid [8], the viscous effects [9], and the initial condition effects [10] on the oscillation. A relationship between Ohnesorge number $(\mathrm{Oh}$, a dimensionless number that relates the viscous forces to inertial and surface tension forces) and critical damping (corresponding to the shortest coalescence time), for a droplet i.e. $0.71<O h<0.76$ was found [11] in the early 1990s. Stover et. al[12] were the first to experimentally study the microbubble oscillation and showed the effects of liquid viscosity and surface tension on the decay of the damped oscillation. Recently, a benchmark study[13] exhibited the different morphological evolutions in the global coalescence from two equal-size spherical droplets to a stable single droplet with minimal surface energy when the viscosity ratio of two-fluids is either low or high. In spite of the efforts in the past one and half centuries, so far the underlying of self-oscillation is still in its infant stage.

Due to the delicate and ephemeral nature of microbubble coalescence, experimental exploration of the fundamental physics is still exceptionally challenging. Whereas numerical 
simulation provides a unique and powerful capability to characterize the underlying mechanics of microbubble coalescence through parameterizations and classification. Among different numerical schemes, the kinetic-based lattice Boltzmann method (LBM)[14, 15] has demonstrated its physical and computational advantages to simulate multiphase flows[16, 17]. In the past three decades, several multiphase models using LBM have been developed, including the color fluid model [18], the pseudo-potential model [19], the mean-field model et $a l .[20]$, the phase-field model [21] based on the free-energy theory [22], and the entropic LBM [23]. These models have been continuously refined and applied to simulate many multiphase flow problems, (see general LBM reviews [16, 17] and specific multiphase LBM reviews [24, 25], and therein references). We employ the free-energy modeling approach that has been continuously developed and refined in the last 10 years by the Lee group [26, 27, 28, 29] and it has been shown that the parasitic current (an artificial velocity field caused by discretization errors in the simulation of multiphase flows) has been eliminated [28] and large density gradients of up to 1000 [27] across the interface can be handled. As part of the continuous effort to unveil the underlying physics of microbubble coalescence, in this work, we explore the mechanism of damped oscillation in microbubble coalescence from initially two touched equal size microbubbles to finally one coalesced bubble with minimum surface area. The focus is to address when and how a damped oscillation occurs during a coalescence process in terms of the $O h$ number.

\section{Lattice Boltzmann Modeling for Fluid-gas Flows}

The detail formulation of the lattice Boltzmann model can be found in the paper[30]. For the sake of comprehension and completion, we concisely introduce the main idea and major equations here. A diffuse interface is applied to separate phases in the modelling of multiphase flow. There are three governing equations including continuity equation, pressure evolution and momentum equation as follows. The continuity equation can be written as 
the Cahn-Hilliard equation.

$$
\begin{gathered}
\partial C / \partial t+\mathbf{u} \cdot \nabla C=\nabla \cdot(M \nabla \mu) \\
\partial p_{1} / \partial t+\rho c_{s}^{2} \nabla \cdot \mathbf{u}=0 \\
\rho(\partial \mathbf{u} / \partial t+\mathbf{u} \cdot \nabla \mathbf{u})=-\nabla p_{1}+\mu \nabla C+\nabla \cdot \eta\left(\nabla \mathbf{u}+(\nabla \mathbf{u})^{T}\right)
\end{gathered}
$$

Here $M(>0)$ is the mobility [31] and $C$ is the composition, $\mu$ is the chemical potential defined as $\mu=\mu_{0}-\kappa \nabla^{2} C$ in which $\mu_{0}$ is the classical part of the chemical potential. In the vicinity of the critical point, simplification of van der Waals equation of state can be made[32] for the control of interface thickness and surface tension at equilibrium. In this case, we assume that the energy $E_{0}$ takes a form [33ł of $E_{0}=\beta C^{2}(C-1)^{2}$ with $\beta$ being a constant. As a result, $\mu_{0}=\partial E_{0} / \partial C=2 \beta C(C-1)(2 C-1)$. In an interface at equilibrium, the interface profile is $C(z)=0.5+0.5 \tanh (2 z / D)$ where $z$ is the distance normal to the interface and $D$ is the numerical interface thickness, which is chosen based on accuracy and stability. Given $\mathrm{D}$ and $\beta$, one can compute the gradient parameter $\kappa=\beta D^{2} / 8$ and the surface tension force $\sigma=\sqrt{2 \kappa \beta} / 6$. For a binary flow, we introduce the intermolecular force [28] as $\mathbf{F}=\frac{1}{3} \nabla \rho c^{2}-\nabla p_{1}-C \nabla \mu$ where $p_{1}$ is the hydrodynamic pressure, whereas the thermodynamic pressure $p_{0}$ is defined by $p_{0}=C \partial E_{0} / \partial C-E_{0}=\beta C^{2}(C-1)(3 C-1)$. The total pressure is $p \bumpeq p_{0}+p_{1}-\kappa C \nabla^{2} C+\kappa|\nabla C|^{2} / 2$.

The lattice Boltzmann equation (LBE) (before the time discretization) including the intermolecular force reads [20]

$$
\partial f_{\alpha} / \partial t+\mathbf{e}_{\alpha} \cdot \nabla f_{\alpha}=-\left(f_{\alpha}-f_{\alpha}^{e q}\right) / \lambda+\frac{3}{c^{2}}\left(\mathbf{e}_{\alpha}-\mathbf{u}\right) \cdot \mathbf{F} f_{\alpha}^{e q}
$$

where $f_{\alpha}$ is the particle distribution function with discrete molecular velocity $\mathbf{e}_{\alpha}$ along the $\alpha$-th direction and $\lambda$ is the relaxation time related to the kinematic viscosity $\nu=\frac{1}{3} c^{2} \lambda$. The equilibrium distribution function is defined as $f_{\alpha}^{e q}=\rho \omega_{\alpha}\left[1+3\left(\mathbf{e}_{\alpha} \cdot \mathbf{u}\right) / c^{2}+9\left(\mathbf{e}_{\alpha} \cdot \mathbf{u}\right)^{2} /\left(2 c^{4}\right)-\right.$ 
$\left.3 \mathbf{u}^{2} /\left(2 c^{2}\right)\right]$ where $\omega_{\alpha}$ is the weight associated with a particular discretized velocity $\mathbf{e}_{\alpha}, \rho$ and $\mathbf{u}$ are macroscopic density and velocity respectively, and $c=\delta x / \delta t=1$ in lattice units (i.e., $\delta t=\delta x=1)$.

Defining a new particle distribution function $g_{\alpha}=\frac{1}{3} f_{\alpha} c^{2}+\left(p_{1}-\frac{1}{3} \rho c^{2}\right) \Gamma_{\alpha}(0)$ in which $\Gamma_{\alpha}(\mathbf{u})=f_{\alpha}^{e q} / \rho$ and taking the total derivative $D_{t}=\partial_{t}+\mathbf{e}_{\alpha} \cdot \nabla$ of $g_{\alpha}$ result in

$$
\partial g_{\alpha} / \partial t+\mathbf{e}_{\alpha} \cdot \nabla g_{\alpha}=-\left(g_{\alpha}-g_{\alpha}^{e q}\right) / \lambda+\left(\mathbf{e}_{\alpha}-\mathbf{u}\right) \cdot\left[\frac{1}{3} \nabla \rho c^{2}\left(\Gamma_{\alpha}-\Gamma_{\alpha}(0)\right)-C \nabla \mu \Gamma_{\alpha}\right]
$$

where the new equilibrium $g_{\alpha}^{e q}$ is $g_{\alpha}^{e q}=\omega_{\alpha}\left[p_{1}+\rho\left((\mathbf{e} \cdot \mathbf{u})+3\left(\mathbf{e}_{\alpha} \cdot \mathbf{u}\right)^{2} / 2 c^{2}-u^{2}\right)\right]$

Discretizing Eq. (5) along characteristics over the time step $\delta t$, we obtain the LBE for $g_{\alpha}$

$$
\begin{aligned}
\bar{g}_{\alpha}\left(\mathbf{x}+\mathbf{e}_{\alpha} \delta t, t+\delta t\right)= & \bar{g}_{\alpha}(\mathbf{x}, t)-\frac{1}{\tau+0.5}\left(\bar{g}_{\alpha}-\bar{g}_{\alpha}^{e q}\right) \mid(\mathbf{x}, t)+ \\
& \left.\left(\mathbf{e}_{\alpha}-\mathbf{u}\right) \cdot\left[\frac{1}{3} \delta t \nabla^{M D} \rho c^{2}\left(\Gamma_{\alpha}(\mathbf{u})-\Gamma_{\alpha}(0)\right)-C \delta t \nabla^{M D} \mu \Gamma_{\alpha}\right]\right|_{(\mathbf{x}, t)}
\end{aligned}
$$

where $\nabla^{M D}$ and $\nabla^{C D}$ are referred to mixed difference approximation and central difference approximation respectively [29] and $\tau(=\lambda / \delta t)$ is the non-dimensional relaxation time. In Eq. (6), the modified particle distribution function $\bar{g}_{\alpha}$ and the equilibrium distribution function $\bar{g}_{\alpha}^{e q}$ are introduced to facilitate computation

$$
\begin{gathered}
\bar{g}_{\alpha}=g_{\alpha}+\frac{1}{2 \pi}\left(g_{\alpha}-g_{\alpha}^{e q}\right)-\frac{1}{2} \delta t\left(\mathbf{e}_{\alpha}-\mathbf{u}\right) \cdot\left[\frac{1}{3} \nabla^{C D} \rho c^{2} C\left(\Gamma_{\alpha}(\mathbf{u})-\Gamma_{\alpha}(0)\right)-C \nabla^{C D} \mu \Gamma_{\alpha}\right] \\
\bar{g}_{\alpha}^{e q}=g_{\alpha}^{e q}-\frac{1}{2} \delta t\left(\mathbf{e}_{\alpha}-\mathbf{u}\right) \cdot\left[\frac{1}{3} \nabla^{C D} \rho c^{2}\left(\Gamma_{\alpha}(\mathbf{u})-\Gamma_{\alpha}(0)\right)-C \nabla^{C D} \mu \Gamma_{\alpha}\right]
\end{gathered}
$$

The momentum and hydrodynamic pressure are the zeroth and first-order moment of $\bar{g}_{\alpha}$, computed as $\rho u=\frac{3}{c^{2}} \sum \mathbf{e}_{\alpha} \bar{g}_{\alpha}-\frac{\delta t}{2} C \nabla^{C D} \mu$ and $p_{1}=\sum \bar{g}_{\alpha}+\frac{\delta t}{6} \mathbf{u} \cdot \nabla^{C D} \rho c^{2}$

For the transformation of the composition $C$, a second distribution function is introduced in a simple format of $h_{\alpha}=(C / \rho) f_{\alpha}$ and $h_{\alpha}^{e q}=(C / \rho) f_{\alpha}^{e q}$. Similarly, taking the total derivative 
$D_{t}$ of $h_{\alpha}$ and utilizing Eq. (1) yield

$$
\begin{aligned}
\bar{h}_{\alpha}\left(\mathbf{x}+\mathbf{e}_{\alpha} \delta t, t+\delta t\right)= & \bar{h}(\mathbf{x}, t)-\frac{\bar{h}_{\alpha}-\left.\bar{h}_{\alpha}^{e q}\right|_{(\mathbf{x}, t)}}{\tau+0.5}+\delta t\left(\mathbf{e}_{\alpha}-\mathbf{u}\right) \cdot\left[\nabla^{M D} C-\frac{3 C}{\rho c^{2}}\left(\nabla^{M D} p_{1}\right.\right. \\
& \left.\left.+C \nabla^{M D} \mu\right)\right]\left.\Gamma_{\alpha}\right|_{(\mathbf{x}, t)}+\left.\delta t M \nabla^{2} \mu \Gamma_{\alpha}\right|_{(\mathbf{x}, t)}
\end{aligned}
$$

where the modified particle distribution function $\bar{h}_{\alpha}$ and $\bar{h}_{\alpha}^{e q}$ are defined as [29]

$$
\begin{gathered}
\bar{h}_{\alpha}=h_{\alpha}+\frac{1}{2 \tau}\left(h_{\alpha}-h_{\alpha}^{e q}\right)-\frac{\delta t}{2}\left(\mathbf{e}_{\alpha}-\mathbf{u}\right) \cdot\left[\nabla^{C D} C-\frac{3 C}{\rho c^{2}}\left(\nabla^{C D} p_{1}+C \nabla^{C D} \mu\right)\right] \Gamma_{\alpha} \\
\bar{h}_{\alpha}^{e q}=h_{\alpha}^{e q}-\frac{\delta t}{2}\left(\mathbf{e}_{\alpha}-\mathbf{u}\right) \cdot\left[\nabla^{C D} C-\frac{3 C}{\rho c^{2}}\left(\nabla^{C D} p_{1}+C \nabla^{C D} \mu\right)\right] \Gamma_{\alpha}
\end{gathered}
$$

The composition $C$ is the zeroth-order moment of $\bar{h}_{\alpha}$ computed as $C=\sum_{\alpha} \bar{h}_{\alpha}+0.5 \delta t M \nabla^{2} \mu$. The density $\rho$ and the dimensionless relaxation frequency $(1 / \tau)$ are taken as linear functions of the composition by $\rho(C)=C \rho_{1}+(1-C) \rho_{2}$ and $1 / \mathcal{\tau}(C)=C / \tau_{1}+(1-C) / \tau_{2}$.

\section{Numerical Study}

The objective of the current study is to address two questions for two equal-size microbubbles that coalesce. First, when does self-oscillation occur? Second, how is a damped oscillation driven and developed?

\subsection{Computational set-up}

As schematized in Fig. 1(a), two equal-size microbubbles are set in the center of a microfluidic channel with a square domain of $100^{2}\left(\mu m^{2}\right)$. Subscripts of "h" and "l" denote the heavy (liquid) and light (gas) fluid respectively. The gas is fixed to air with density $\rho_{l}=1.28 \mathrm{~kg} / \mathrm{m}^{3}$ and dynamic viscosity $\eta_{l}=1.74 \times 10^{-5} \mathrm{~kg} /(\mathrm{m} \cdot \mathrm{s})$. The density of liquid is fixed as $\rho_{h}=1840 \mathrm{~kg} / \mathrm{m}^{3}$ but varies the viscosity $\eta_{h}$ from 0.002 to $0.08 \mathrm{~kg} /(\mathrm{m} \cdot \mathrm{s})$. Constant surface tension, $\sigma=7.3 \times 10^{-2} \mathrm{~N} / \mathrm{m}$, is assumed. This physical setting results in a range of $O h\left(\equiv \eta_{h} / \sqrt{\rho_{h} \sigma R}\right)$ numbers from 0.039 to 1.543 . The initial bubble radius $R$ is $20 \mu m$. $D_{x}$ and $D_{y}$ are the distances between the bubble edge and the mass center $O$ in the horizontal 


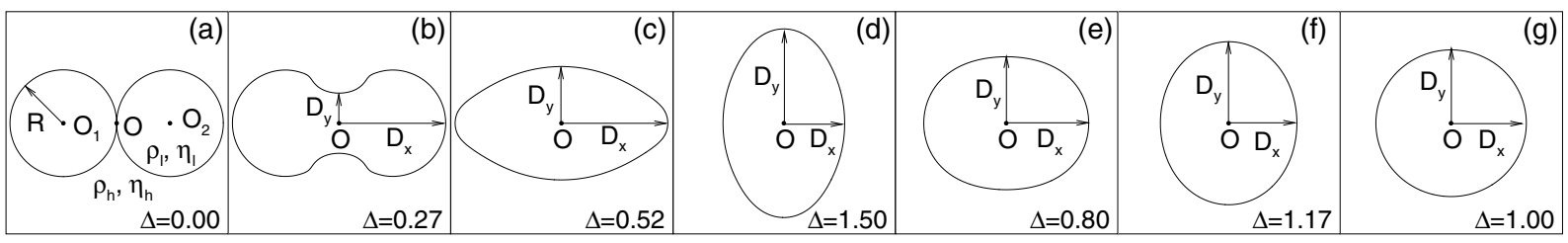

Figure 1: A typical microbubble coalescence process with damped oscillation in a microfluidic channel from (a)initially touched bubbles to (b) neck bridge evolution through (c)-(f) oscillation with damping axis ratio toward $(\mathrm{g})$ a coalesced bubble with the minimum surface area. $\rho_{l} / \rho_{h}$ and $\eta_{l} / \eta_{h}$ are density and dynamic viscosity of gas/liquid respectively. $D_{x}$ and $D_{y}$ are the distances between bubble edge and the mass center $O$ in the horizontal and vertical directions respectively, and $\Delta\left(=D_{y} / D_{x}\right)$ is the shape factor of the coalescing bubble.

and vertical directions respectively. A shape factor, defined as $\Delta=D_{y} / D_{x}$, is to track the interface of the coalescing bubble, starting from 0 (initial touched bubble) and ending at 1 (single coalesced bubble), as schematized in Fig. 1. In our previous study[34], a switching of the major axis of the coalescing bubble between the horizontal and vertical directions was observed, when the $O h$ number is relatively small, in the post-coalescence corresponding to the period from (c) to (g) in Fig. 1. Since the shape factor $\Delta$ is reducing, this phenomenon is called damped oscillation. In general, the bubble coalescence can be categorized into three types of damping: underdamping (with a visible oscillation of $\Delta$ ), overdamping (invisible oscillation of $\Delta$ ) and critical damping (invisible oscillation with the shortest time to reach $\Delta=1$ ). The in-house, GPU-accelerated LBM code based on the free-energy model is used for all the simulation in this work. The reliability of this LBM model has been demonstrated in some application studies $[30,35,34]$ previously through comparisons with analytical solutions and experimental/computational results. The spatial resolution was selected $600^{2}$ through a convergence check[34]. The periodic boundary condition is applied in both directions.

\subsection{Numerical Results}

In this part, we explore when and how a damped oscillation occurs during a coalescence through a systematic study. Table 1 shows the sixteen cases with identical physical and computational conditions except for the fluid viscosity $\eta_{h}$ and corresponding different $O h$ numbers.

Figure 2 shows the time evaluation of the shape factor, $\Delta\left(=D_{y} / D_{x}\right)$, of 5 representative 


\begin{tabular}{|c|c|c|c|c|c|c|c|c|c|c|c|c|c|c|c|c|}
\hline Case & 1 & 2 & 3 & 4 & 5 & 6 & 7 & 8 & 9 & 10 & 11 & 12 & 13 & 14 & 15 & 16 \\
\hline$\eta_{h} \times 10^{3}(\mathrm{~kg} / \mathrm{m} \cdot \mathrm{s})$ & 2.0 & 3.5 & 6.5 & 9.2 & 13.7 & 18.0 & 21.0 & 24.7 & 27.5 & 35.0 & 40.0 & 45.0 & 50.0 & 55.0 & 70.0 & 80.0 \\
\hline$O h \times 10$ & 0.39 & 0.67 & 1.25 & 1.77 & 2.64 & 3.47 & 4.07 & 4.77 & 5.30 & 6.75 & 7.72 & 8.68 & 9.65 & 10.61 & 13.51 & 15.43 \\
\hline
\end{tabular}

Table 1: Sixteen cases with identical physical and computational conditions except for the fluid viscosity $\eta_{h}$, thus different $O h$ numbers.

cases with $O h=0.039,0.177,0.477,0.675$, and 0.964 . The $O h$ value clearly affects the coalescence style. On one side when $O h<0.477$, the blue lines with solid symbols exhibit damped oscillation, indicating the axis switching of the coalescing bubble between horizontal and vertical directions with reducing the amplitude of $\Delta$ toward a final circular bubble. The smaller the $O h$ number, the stronger the oscillation. On the other side when $O h>0.477$, the green lines with empty symbols show asymptotic growth of the shape factor toward the end of the coalescence when $\Delta=1.0$, implying that the coalescing bubble retains its major axis on the horizontal direction in the entire process of coalescence with no oscillation. In between, $O h=0.477$ serves as the dividing edge for the two distinct coalescence styles.

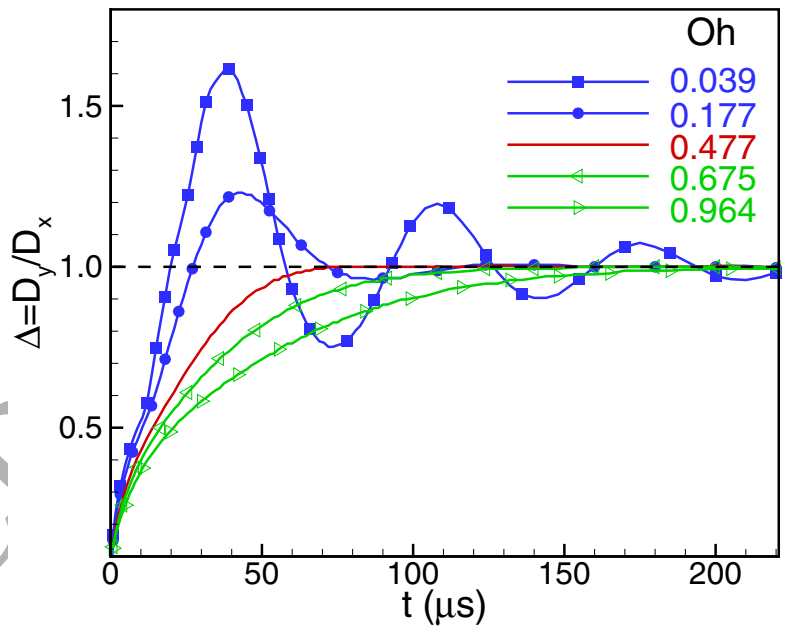

Figure 2: Time evolution of shape factor $\left(\Delta=D_{y} / D_{x}\right)$ of 5 representative cases during bubble coalescence. Two distinct coalescence phenomena, with and without oscillations when $\mathrm{Oh}<0.477$ and $\mathrm{Oh}>0.477$ respectively, are identified. $O h=0.477$ serves as the dividing edge of them.

Another effect of $O h$ numbers on bubble coalescence is the coalescence time, denoted as $T$ in $\mu s$, from two equal, initially-touched bubbles to finally one circular bubble when the minimum surface area, no matter with or without oscillations, as shown in Fig. 3. When the $O h$ number is small (in blue) where damped oscillation is involved, increasing 
the $O h$ number can significantly reduce the coalescence time $T$. Whereas when the $O h$ number is large (in green) with no oscillation, increasing that $O h$ number causes larger T meaning longer coalescence process. For the $O h$ resolution selected in this study, there exists a critical $O h$ number, i.e. 0.477 (in red), that corresponds the shortest coalescence time. If considering a continuous $O h$ range, the critical $O h$ number should be identified in the range from 0.407 to 0.530 . To the authors' best knowledge, such criteria for with and without oscillation of microbubble coalescence is a first-time finding. A similar criterion for droplets were previously discovered, from which the critical $\mathrm{Oh}$ number is believed to be between 0.71 to $0.76[11]$.

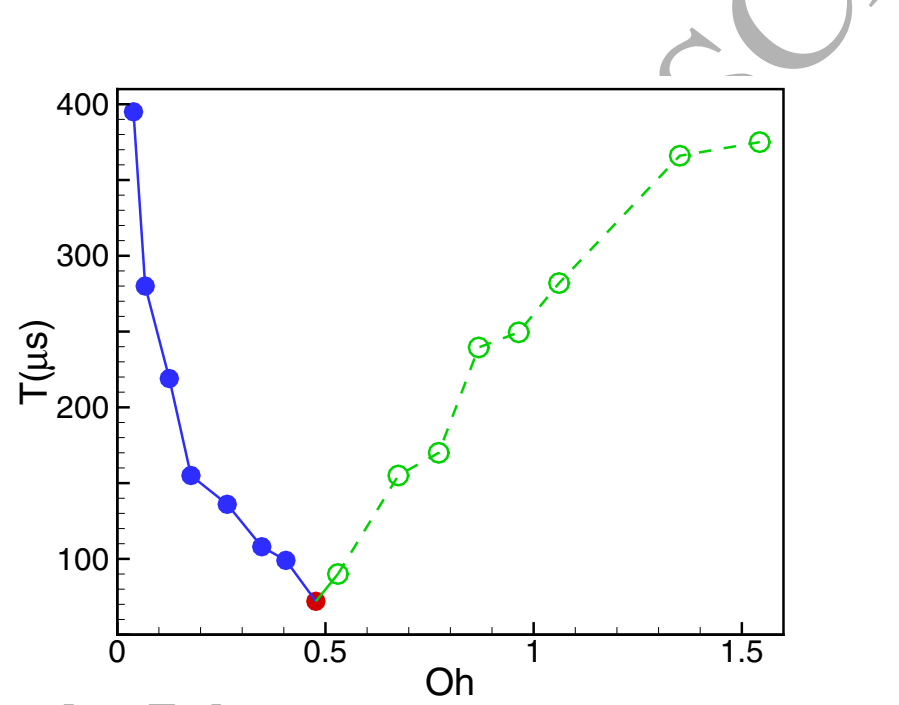

Figure 3: Effects of $O h$ on the bubble coalescence time $T$. Blue/green lines correspond to small/large $O h$ ranges. When $O h$ is small/large, increasing $O h$ reduces/increases coalescence time. The critical $O h(=0.477)$ corresponds to the shortest coalescence time that distinguishes the two distinct coalescence phenomena.

We now explore the mechanism behind these two distinct coalescence phenomena in terms of the competition between driving and resisting forces at different $O h$ ranges. Since, in all the study cases listed in Table 1, only liquid viscosity varies, causing the variation of the Oh number while all other parameters remain the same. We only consider the imbalance of surface tension at interface and viscous resistance from liquid in this part. Surface energy due to the surface tension can be divided into two parts. One is released to produce kinetic energy to drive the coalescence, which can be called useful energy. The other part is dissipated due 
to the viscous effect.

First, we focus on the left side (in blue) in Fig. 3. In this regime, the viscosity is relatively small, thus the coalescence is dominated by surface tension. To better describe the phenomenon, we stick to the top half of the coalescing bubble and the middle point of the interface (called the saddle point). Once the neck is formed, see Fig. 1(b), the surface tension induces a strong acceleration of the saddle point away from the bubble center, conyerting surface energy to kinetic energy. When the interface gets flat, the velocity magnitude of the saddle point reaches the maximum as all of the useful surface energy has been converted to the kinetic energy for the interface motion. Due to the inertia, $D_{y}$ continues to increase and the interface becomes a convex shape, Fig. 1(c), generating surface tension. The velocity of the saddle point slows down as the kinetic energy is being converted to surface energy. When all the useful kinetic energy becomes surface energy, $D_{y}$ reaches its peak and surface tension gets to the maximum Fig. 1(d). The process from (c) to (d) illustrates the switching of the major axis from horizontal to vertical directions. The similar transformation between surface energy and kinetic energy occurs to switch the major axis from vertical to horizontal directions, Fig. 1(d)-(e), in the opposite direction, completing the first cycle of the oscillation. Because of the existence of the energy dissipation, useful energy is losing and the amplitude of $D_{y}$ is reduced when the saddle point is back to its lowest location. Such cycles repeat with smaller and smaller amplitudes till it reaches the final stable coalesced bubble. In Fig. 3 , it is seen that smaller $O h$ results in faster growth and a higher peak of $\Delta$. This is because smaller energy dissipation permits more useful surface energy to drive the bubble to coalesce. If we consider that the surface tension acts as a restoring force, the surrounding fluid acts as a mass, and viscosity damps the motion, the 1-D motion of the saddle point can be modelled as a damped harmonic oscillator [36]

$$
A^{\prime} \rho R^{3} \frac{d^{2} D_{y}^{2}}{d t^{2}}+B^{\prime} \rho \nu R \frac{d D_{y}}{d t}+C^{\prime} \sigma D_{y}=0
$$


where $A^{\prime}, B^{\prime}, C^{\prime}$ are dimensionless geometric parameters to be determined. The damped oscillation of the saddle point can be derived as the solution of the Eq. (12)

$$
D_{y}=A e^{\frac{-B \nu t}{R^{2}}} \sin \left(C \sqrt{\frac{\sigma}{\rho R^{3}}} t\right)
$$

in which $A$ (integral constant to be determined), $B\left(=\frac{B^{\prime}}{2 A^{\prime}}\right.$, and $C\left(=\sqrt{\frac{C^{\prime}}{A^{\prime}}-\frac{B^{\prime 2}}{A^{\prime}} \frac{O h^{2}}{4}}\right)$ correspond to the amplitude coefficient, decay factor, and the oscillation period respectively. We select three representative cases of $O h=0.067,0.125$ and 0.177 and use the numerical results to determine $A, B$, and $C$ respectively with the start point $D_{y} \neq R_{e}$. As shown in Fig. 4, the oscillating trajectories of the saddle point with reduced amplitudes (symbols) are well-captured by the damped harmonic oscillator model (solid lines).

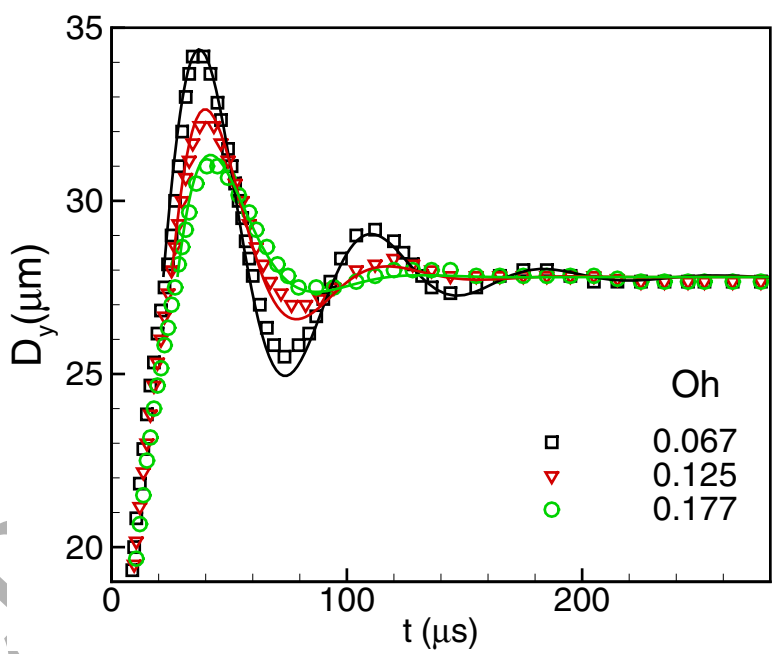

Figure 4: The oscillating trajectories of the saddle point, represented by $D_{y}$, with reduced amplitudes with $O h=0.067$ (black), 0.125 (red), and 0.177(green). Symbols: numerical results; solid lines: analytical solutions of Eq. (13).

Then, we look at the green side in Fig. 3. In this regime, the viscosity is large enough to resist the coalescence. Each of the green lines in Fig. 2 shows a monotonic growth of the shape factor from 0 to 1 , indicating that no oscillation occurs in the coalescence. When $O h$ increases, the coalescence time increases. This is understandable because the larger $O h$ means large resistance thus this slows down the coalescence process. 


\section{Summary \& Future Work}

Using the GPU-accelerated LBM simulation, we are able to systematically investigate the effects of $O h$ number on the global coalescence process in a microchannel. Sixteen cases with the $O h$ number from 0.039 to 1.543 , by varying the liquid viscosity from 0.0020 to $0.08 \mathrm{~kg} /(\mathrm{m} \cdot \mathrm{s})$ while keeping other parameters unchanged, are studied. By tracking the time evolution of the shaper factor $\Delta$, we identified two distinct coalescence phenomena. In the region of $O h<0.477$, damped oscillation is observed. The oscillation is more intensive when $O h$ is smaller, and resulting a longer time to complete the gløbal oscillation. While $O h>0.477$, the shape factor asymptotically increases from 0 /to 1 and the global coalescence time increases with the increase of $O h$. The mechanism behind the different coalescence behavior is explored in terms of the competition of the surface tension driving and viscous resisting. When $O h<0.477$, the viscous force is small thus energy dissipation is insignificant. The bubble coalescence is dominated by the surface tension force. The transformation between the surface energy and kinetic energy with energy dissipation causes the damped oscillation of microbubble coalescence. Smaller Oh number cases possess more useful surface energy to drive stronger oscillation, resulting in longer coalescence time. The damped oscillation can be modeled as a damped harmonic oscillator that has an analytical solution. The numerical simulation has a good agreement with the analytical prediction. Whereas in the range of $O h>0.477$, the viscous force becomes significant. The neck growth is much slower and no overshooting of the bubble interface occurs and the shape factor asymptotically grows from 0 to 1 . It is seen that a larger $O h$ number corresponds with longer coalescence time, implying the role of the viscous effect. In between the two regimes, a critical $O h=(0.477)$ number is identified, corresponding to the smallest coalescence time. It should be noted that the observed critical $O h$ number is based on the discretization of $O h$ in the current study. Strictly speaking, when consider a continuous $O h$ range, the critical Oh number should fall in the range between 0.407 and 0.530 . Such a criterion of with and without damped oscillation determined by $O h$ number are consistent to the drop coalescence 
that has presented in open data. To authors' best knowledge, the finding of the critical $O h$ number that separates the two regimes corresponding to the distinct coalescence behavior for microbubble is believed to be the first. There are further questions to be investigated: (1) what determines the critical $O h$ number? (2) what occurs if the initial two microbubbles are unequal? (3) what will be different if the bubble coalescence occurs on a solid surface? Some of them are being addressed and the results will be presented in near future.

\section{Acknowledgments}

This research is supported by the graduate assistantship from School of Engineering and Technology. And this work used the Extreme Science and Engineering Discovery Environment (XSEDE), which is supported by National Science Foundation grant number ACI-1053575.

\section{References}

\section{References}

[1] T. Tsukada, M. Sato, N. Imaishi, M. Hozawa, K. Fujikawa, A theoretical and experimental study on the oscillation of a hanging drop, Journal of chemical engineering of Japan 20 (1) (1987) 88-93.

[2] D. Gaitan, L. Crum, C. Church, R. Roy, Sonoluminescence and bubble dynamics for a single, stable, cavitation bubble, The Journal of the Acoustical Society of America 91 (6) (1992) 3166-3183.

[3] I. Akhatov, O. Lindau, A. Topolnikov, R. Mettin, N. Vakhitova, W. Lauterborn, Collapse and rebound of a laser-induced cavitation bubble, Physics of Fluids 13 (10) (2001) $2805-2819$. 
[4] S. Karakashev, P. Nguyen, R. Tsekov, M. Hampton, A. Nguyen, Anomalous ion effects on rupture and lifetime of aqueous foam films formed from monovalent salt solutions up to saturation concentration, Langmuir 24 (20) (2008) 11587-11591.

[5] N. Matsuki, S. Ichiba, T. Ishikawa, O. Nagano, M. Takeda, Y. Ujike, T. Yamaguchi, Blood oxygenation using microbubble suspensions, European Biophysics Journal 41 (6) (2012) 571-578.

[6] G. Kaushik, A. Chel, Microbubble technology: emerging field for water treatment, Bubble Science, Engineering \& Technology 5 (1-2) (2014) 33-38.

[7] L. Rayleigh, On the capillary phenomena of jets, Próc. R. Soc. London 29 (196-199) (1879) 71-97.

[8] H. Lamb, Hydrodynamics, Cambridge university press, 1932.

[9] C. Miller, L. Scriven, The oscillations of a fluid droplet immersed in another fluid, Journal of Fluid Mechanics 32 (3) (1968) 417-435.

[10] A. Prosperetti, Free oscillations of drops and bubbles: the initial-value problem, Journal of Fluid Mechanics 100(2) (1980) 333-347.

[11] O. Basaran, Nonlinear oscillations of viscous liquid drops, Journal of Fluid Mechanics $241(1992) 169-198$

[12] R. Stover, C. Tobias, M. Denn, Bubble coalescence dynamics, AIChE J 43 (10) (1997) $2385-2392$.

[13] J. Sprittles, Y. Shikhmurzaev, Coalescence of liquid drops: Different models versus experiment, Physics of Fluids 24 (12) (2012) 122105.

[14] H. Chen, S. Chen, W. Matthaeus, Recovery of the Navier-Stokes equations using latticegas Boltzmann method, Physical Review A 45 (1992) R5339-R5342. 
[15] Y. Qian, D. Dhumieres, P. Lallemand, Lattice Boltzmann model for Navier-Stokes equation, Europhysics Letters 17 (1992) 479-484.

[16] S. Chen, G. Doolen, Lattice Boltzmann method for fluid flows, Annual Review of Fluid Mechanics 30 (1998) 329-364.

[17] C. Aidun, J. Clausen, Lattice-Boltzmann method for complex flows, Annual Review of Fluid Mechanics (2010) 439-472.

[18] K. Andrew K. Gunstensen, H. Daniel H Rothman, S. Zaleski, G. Zanetti, Lattice Boltzmann model of immiscible fluids, Phys. Rev. A 43 (1991) 4320-27.

[19] X. Shan, H. Chen, Lattice Boltzmann model for simulating flows with multiphases and components, Physical Review E 47 (1993) 1815-1819.

[20] X. He, X. Shan, G. Doolen, Discrete Boltzmann equation model for nonideal gases, Physical Review E 57 (1998) R13.

[21] M. Swift, W. Osborn, J. Yeomans, Lattice Boltzmann simulation of nonideal fluids, Phys. Rev. Let. 75 (5) (1995) 830-833.

[22] J. Cahn, J. Hilliard, Free energy of a nonuniform system. I. Interfacial free energy, J. Chem. Phys. 28 (1958) $258-266$.

[23] A. Mazloomi M, S. Chikatamarla, I. Karlin, Entropic lattice Boltzmann method for multiphase flows, Phys. Rev. Lett. 114 (2015) 174502.

[24] L. Chen, Q. Qinjun Kang, Y. Mua, Y.-L. He, W.-Q. Tao, A critical review of the pseudopotential multiphase lattice Boltzmann model: Methods and applications, International Journal of Heat and Mass Transfer 76 (2014) 210 - 236.

[25] K. Connington, T. Lee, A review of spurious currents in the lattice Boltzmann method for multiphase flows, Journal of Mechanical Science and Technology 26 (12) (2012) $3857-3863$. 
[26] T. Lee, C.-L. Lin, A stable discretization of the lattice Boltzmann equation for simulation of incompressible two-phase flows at high density ratio, J. Comp. Phys. 206 (10) (2005) $16-47$.

[27] T. Lee, P. Fischer, Eliminating parasitic currents in the lattice Boltzmann equation method for nonideal gases, Physical Review E 74 (4) (2006) 046709.

[28] T. Lee, Effects of incompressibility on the elimination of parasitic currents in the lattice Boltzmann equation method for binary fluids, Computers and Mathematics with Applications 58 (2009) $987-994$.

[29] T. Lee, L. Liu, Lattice Boltzmann simulations of micron-scale drop impact on dry surfaces, J Comp Phys 229 (2010) 8045-8063.

[30] R. Chen, H. Yu, L. Zhu, L. Taehun, R. Patil, Spatial and temporal scaling of unequal microbubble coalescence, The AIChE Journal 63 (2017) 1441 - 1450.

[31] M. o. Carpinlioğlu, M. Gündoğdu, A critical review on pulsatile pipe flow studies directing towards future research topics, Flow Meas. Instrum. 12 (3) (2001) 163-174.

[32] J. Rowlinson, B. Widom, Molecular Theory of Capillarity, Oxford Univ. Press, Oxford, UK, 1989.

[33] D. Jamet, O. Lebaigue, N. Coutris, J. Delhaye, The second gradient method for the direct numerical simulation of liquid-vapor flows with phase change, Journal of Computational Physics 169 (2) (2001) 624-651.

[34] R. Chen, H. Yu, L. Zhu, General power-law temporal scaling for unequal microbubble coalescence, The AIChE JournalSubmitted.

[35] R. Chen, H. Yu, L. Zhu, Effects of initial conditions on the coalescence of micro-bubbles, Proceedings of the Institution of Mechanical Engineers, Part C: Journal of Mechanical Engineering Science (2017) 0954406217742941. 
[36] R. Stover, Bubble coalescence dynamics and supersaturation in electrolytic gas evolution, Ph.D. thesis, Lawrence Berkeley National Laboratory (1996).

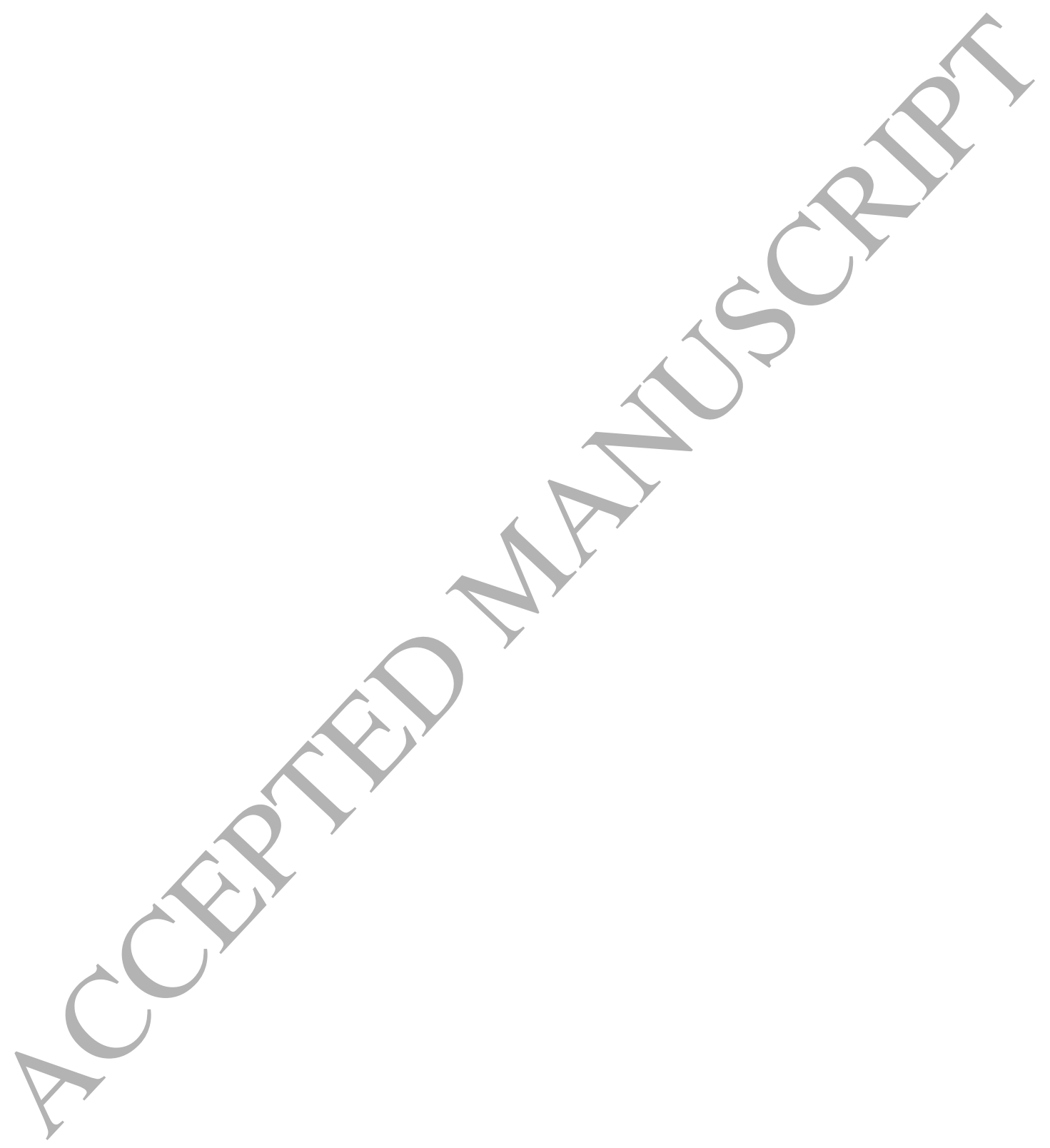

